\title{
Melphalan inhibits adenoma development through modulating the expression of K-ras-specific markers in K-ras Tg mice
}

\author{
SOJUNG LEE ${ }^{1}$, HEESOOK CHOI ${ }^{1}$, EUNJIN KIM ${ }^{1}$, HEEJONG KIM ${ }^{1}$, YOUNG-HO PARK ${ }^{2}$, DAE-YEUL YU ${ }^{2}$, \\ SEOK-JOO YOON ${ }^{3}$, JINMAN KIM ${ }^{4}$, YHUNYHONG SHEEN ${ }^{5}$, SUE-NIE PARK ${ }^{6}$ and DO-YOUNG YOON ${ }^{1}$ \\ ${ }^{1}$ Department of Bioscience and Biotechnology, Bio/Molecular Informatics Center, Konkuk University, Hwayang-dong 1 , \\ Gwangjin-gu, Seoul 143-701; ${ }^{2}$ Animal Disease Model Research Center, Korea Research Institute of Bioscience \\ and Biotechnology; ${ }^{3}$ Korea Institute of Toxicology, Daejeon; ${ }^{4}$ Department of Pathology, Chungnan National \\ University College of Medicine, Daejeon 303-131; ${ }^{5}$ Department of Pharmacy, Ewha Womans University, Seoul; \\ ${ }^{6}$ Division of Toxicological Research, National Institute of Food and Drug Safety Evaluation, Korea Food and \\ Drug Administration, No. 194 Tongil-Ro, Eunpyung-Gu, Seoul 122-704, Korea
}

Received January 20, 2010; Accepted April 14, 2010

DOI: 10.3892/ijo_00000670

\begin{abstract}
In previous research, we focused on the discovery of K-ras biomarkers, and effects of genotoxic carcinogens on their expression were investigated in this study. It is wellknown that mutated K-ras gene is involved in $~ 30 \%$ of human cancers such as lung cancer. To search for K-ras oncogene-induced modulators in lung tissues of K-ras transgenic mice, we analyzed K-ras-specific genes and proteins related to cancer development, signal transduction, inflammation as well as tumor suppression in a previous study. In this study, we investigated the modulating effects of genotoxic carcinogen treatment on expression of K-rasdependent modulated genes and proteins in lung tissues of $\mathrm{K}$-ras $\mathrm{Tg}$ mice. In order to evaluate candidate $\mathrm{K}$-ras markers modulated by genotoxic stress and to investigate whether a genotoxic carcinogen would enhance or inhibit carcinogenesis in lung tissues of the $\mathrm{K}$-ras $\mathrm{Tg}$ mice, the anti-cancer drug melphalan was intraperitoneally injected into K-ras $\mathrm{Tg}$ mice every two days for four weeks. RT-qPCR and proteomics analyses were performed in order to confirm whether K-ras-specific biomarkers would be modulated by melphalan treatment in K-ras Tg mice. The decreased adenomas were histopathologically observed and K-ras
\end{abstract}

Correspondence to: Dr Do-Young Yoon, Department of Bioscience and Biotechnology, Bio/Molecular Informatics Center, Konkuk University, Hwayang-dong 1, Gwangjin-gu, Seoul 143-701, Korea

E-mail: ydy4218@konkuk.ac.kr

Dr Sue-Nie Park, Division of Toxicological Research, National Institute of Food and Drug Safety Evaluation, Korea Food and Drug Administration, No. 194 Tongil-Ro, Eunpyung-Gu, Seoul 122-704, Korea

E-mail: suenie@kfda.go.kr

Key words: proteomics, K-ras oncogene, melphalan, Tg mouse, cancer expression was suppressed in melphalan-treated $\mathrm{K}$-ras $\mathrm{Tg}$ mice. Melphalan also recovered the expression of K-rasdependent modulated biomarkers. These results suggest that melphalan inhibits carcinogenesis via modulating K-rasspecific genes and proteins expressed in the lung tissues of $\mathrm{K}$-ras $\mathrm{Tg}$ mice.

\section{Introduction}

Lung cancer is a major cause of cancer deaths worldwide regardless of gender (1). Activation of the ras gene family (H-, K- and N-ras) has been detected in lung tumors and preneoplastic lesions $(2,3)$. Activating mutations in K-ras oncogenes are one of the most common genetic alterations in human lung cancer (4). Mutations of K-ras gene have been demonstrated in $40-50 \%$ of lung cancer and activating mutations of the K-ras oncogene are found in one-quarter to one-half of human lung adenocarcinomas. The majority of $\mathrm{K}$-ras genetic alterations are guanine to thymidine point mutations in codon 12 (i.e. G12D), resulting in the change of the encoded amino acid from glycine $(G)$ to aspartic acid (D) (4). Oncogenic K-ras stimulates activation of the Akt/mTOR pathway, which contributes to the development of lung tumors. Also, activation of K-ras in the mouse lung generates an inflammatory process. In mice genetically engineered to express mutant K-ras, mTOR inhibition has been shown to reduce inflammatory processes in the lung $(5,6)$. Some studies have proposed that K-ras mutations play a significant role in the onset and progression of lung cancer (7) and there is powerful evidence that environmental factors such as cigarette smoke, air pollution and occupational exposure to industrial chemicals contribute to lung carcinogenesis (8). In a previous study using Omics approaches, various transcripts and proteins related to cancer development, signal transduction, migration and cancer cell adhesion were modulated by K-ras oncogene in the lung tissues of K-ras transgenic mice (9). Genes related to cell cycle (Cdc37), cancer cell adhesion (glycam 1, integrin $\alpha 8$, integrin $\alpha \mathrm{X}$ and Clec4n), signal transduction (Tlr2, IL-33 and 
Table I. Primer pairs used for real-time qPCR analysis of gene expression.

\begin{tabular}{lll}
\hline Gene & \multicolumn{1}{c}{ Forward primer } & \multicolumn{1}{c}{ Reverse primer } \\
\hline K-ras oncogene & 5'-TGGAGCTGGTGGCGTAGGCA-3' & 5'-AGGCATCGTCAACACCCTGTC-3' \\
Myomesin 2 & 5'-GTCTCAAGCGGCTTCTTACG-3' & 5'-ATTGTCTTCCACCGTCTGCT-3' \\
Sarcolipin & 5'-AATACTGAGGGGCCATGCTA-3' & 5'-GGTGTGTCAGGCATTGTGAG-3' \\
Actin $\alpha$, cardiac (Atct) & 5'-CCAACCGTGAGAAGATGACC-3' & 5'-TGAGTTACACCATCGCCAGA-3' \\
Protein kinase, & 5'-CAACCACCCGAACCTATGAC-3' & 5'-TGGGGATCCAATCTCTTCAG-3' \\
cGMP-dependent, type II & & \\
Titin & 5'-GGCGTGCAGATCTCCTTTAG-3' & 5'-CTGTCTCGGCTGTCACAAGA-3' \\
Troponin T2, cardiac & 5'-GTGGTGGAGGAGTACGAGGA-3' & 5'-GGCTTCTTCATCAGGACCAA-3' \\
Glycoprotien m6a (Gpm6a) & 5'-CCAGGACTGCTGGAGACACA-3' & 5'-AGCCCCAGTTGTGAAGAAAC-3' \\
Cd200 receptor 4 (Cd200r4) & 5'-GCTCTGCTCTGCTGCTTTTC-3' & 5'-AGGTGATGTTCCTGCCCAAG-3' \\
Calnexin & 5'-TGGTTACTGTGTTTGCTGCTG-3' & 5'-AGGAGTGCTGGCATCTGATTT-3' \\
Destrin & 5'-AGTTCAGGTTGCGGATGAAGT-3' & 5'-TGCACTTTTTGTCTGCACTG-3' \\
Gelsolin & 5'-TATCAGTGGTGTGGCTCTGG-3' & 5'-CTTCTGGCTCTCCTCCCTCT-3' \\
Histone H1 & 5'-AGGTCGGTGGCTTTCAAGAA-3' & 5'-GTGGCTTTGGGTTTCTTGC-3' \\
Cofilin 1 & 5'-GACGACCCCTACACCACTTTT-3' & 5'-TCCTCCTTCTTGCTCTCCTT-3' \\
Nucleolar protein 3 (Nol-3) & 5'-CAAACAGTGCGCATGCCAGA-3' & 5'-ACATGTGGTCCCTGAACTGG-3' \\
Vimentin & 5'-ATTTCTCTGCCTCTGCCAAC-3' & 5'-CCTGTCCATCTCTGGTCTCAA-3' \\
GDI $\alpha$ & 5'-GGTGATCTGGAGAGCTTCAA-3' & 5'-TGACCCCTTTCCTGTATGTG-3' \\
Serine peptidase inhibitor & 5'-AAATCCCAAACCTGCTACCC-3' & 5'-CGAGTTCACACGGAAAGGAT-3' \\
clade B, member 1 (Serpinb1) & & \\
\hline & & \\
\hline
\end{tabular}

Ccbp2), migration [Ccr1, Ccl6 and diaphorase 1 (Cyb5r3)], and cancer development (epiregulin) were suggested as putative prognostic biomarkers for cancer in K-ras $\mathrm{Tg}$ mice. In the present study, we elucidated the effect of melphalan, 4-[bis(2-chloroethyl) amino]-1-phenylalanine, on the expression of K-ras-modulated biomarkers in K-ras Tg mice. The anti-cancer agent melphalan is one of the most useful clinical agents used in the treatment of patients with multiple myeloma and known as a human carcinogen (10). Melphalan is associated with increased repair of DNA damage and induces apoptosis of cancer cells or myeloma cells as a DNA-damaging chemotherapeutic agent $(11,12)$. It is also reported that melphalan augmented TRAIL-induced apoptosis in certain lines and patients treated with melphalan for breast cancer, ovarian cancer and multiple myeloma (bone-marrow cancer) had an increased risk of leukemia (13). In a number of investigations sufficient evidence for the carcinogenicity of melphalan in experimental animals was shown. When administered by intraperitoneal injection, melphalan caused lymphosarcoma, lung tumors in mice and peritoneal sarcoma in rats $(14,15)$. In this study, we investigated whether K-ras-specific biomarkers would be modulated by melphalan, and whether melphalan would exert an influence on a genotoxic carcinogenic or an anti-cancer effect in lung tissues of K-ras transgenic mice. The fourweek melphalan treatment of K-ras transgenic mice modulated the up- and down-regulated K-ras biomarkers and seemed to exhibit an anti-cancer effect. These data provide some genotoxic biomarkers and molecular basis for understanding biological properties of lung cancer and the mechanisms of K-ras-induced tumorigenesis.

\section{Materials and methods}

Generation of K-ras transgenic mice and melphalan treatment. $\mathrm{K}-\mathrm{ras}^{\mathrm{G} 12 \mathrm{D}} \mathrm{Tg}$ mice were established by the method of DNA microinjection into embryos of BDF1 mice as previously described (16). Mutant K-ras ${ }^{\mathrm{G} 12 \mathrm{D}}$ gene expression vector carrying human surfactant protein $\mathrm{C}$ (SPC) promoter was used in order to regulate mutant K-ras gene to be expressed in the lung. These mice were obtained for modulator analysis by K-ras mutant in mouse model of lung cancer. Male and female $\mathrm{K}-\mathrm{ras}^{\mathrm{G} 12 \mathrm{D}} \mathrm{Tg}$ mice (five weeks old) and non- $\mathrm{Tg}$ littermates were injected intraperitoneally with $0.6 \mathrm{mg} / \mathrm{kg}$ of melphalan (Sigma, St. Louis, MO) for four weeks at two-day intervals. After the four-week treatment, each five K-ras $\mathrm{Tg}$ and non-Tg in injection groups and three K-ras Tg and non$\mathrm{Tg}$ mice of each control groups were sacrificed and used for analysis of $\mathrm{K}$-ras biomarkers.

Histopathological examination. The melphalan-treated tissue samples were fixed in $10 \%$ neutral buffered formalin, embedded in paraffin, sectioned and stained with hematoxylin and eosin according to standard methods. The histopathological diagnoses were based on the criteria described by Frith and Ward (17). 
Proteomics analysis. Proteomic approach for protein-profiling modulated by melphalan was performed in melphalan-treated $\mathrm{K}$-ras Tg mice. Details of experimental procedure have been published previously (9). Protein identification was performed by using Spectrum Mill Proteomics Workbench Version A.03.03 (Agilent Technologies). Data files were extracted using Spectrum Mill Data Extractor. A semiquantitative analysis of protein profile data was performed by comparing the total peptide intensity with the peptides of an identified protein. The total peptide intensity was obtained by summing up the peptide intensities of the peptide hits for the protein. False positive rates were calculated as described previously (18).

Quantitative real-time PCR. Total RNAs were isolated from the lung tissue of K-ras oncogene transgenic mice and $2 \mu \mathrm{l}$ of 25X-diluted cDNA product from total RNA by M-MuLV reverse transcriptase (New England Biolabs, Beverly, MA) was used as template. Real-time PCR was performed with relative quantification protocol on a Chromo 4 real-time PCR system (Bio-Rad, Hercules, CA), using $\mathrm{iQ}^{\mathrm{TM}} \mathrm{SYBR}^{{ }^{\circledR}}$-Green Supermix (Bio-Rad) for amplification detection. Details of experimental procedure have been published previously (9). All target genes were normalized to the house-keeping gene, $\beta$-actin, using primers (5'-CTGGGACGATATGGAGA AGA-3' as sense primer, 3'-AGAGGCATACAGGGACA ACA-5' as antisense primer) and compared to normal mouse of the appropriate strain for relative expression values. Each sample was run in triplicate and fold changes represent the ratio of $\mathrm{K}$-ras $\mathrm{Tg}$ mice to control (non-Tg mice). Also, the expression ratio of melphalan-treated non-Tg mice to control and that of melphalan-treated K-ras Tg mice to K-ras Tg mice were represented as fold difference. However, melphalan$\mathrm{K}$-ras data were ultimately revealed as melphalan-K-ras/ $\mathrm{K}$-ras in graphs and total data were made of graph on the basis of non-Tg mice, setting at 1.0. Also, real-time qPCR was performed to investigate expression change of K-ras oncogene by melphalan treatment in K-ras Tg mice. The sequences of the primers used in this experiment were listed previously (9) and those of new primers are in Table I.

Statistical analysis. Results are presented as mean \pm SD. Comparisons between $\mathrm{K}$-ras $\mathrm{Tg}$ and mel-K-ras $\mathrm{Tg}$ groups were performed by Student's t-test. $\mathrm{P}<0.05$ and $\mathrm{P}<0.01$ were considered significant.

\section{Results}

Histopathological examination and expression of $K$-ras by melphalan. In our previous study, several alveolar/bronchiolar hyperplasias and adenomas were histopathologically observed in each lobe of the lung in the K-ras Tg mice (9). By using genotyping, total $16 \mathrm{~K}-$ ras $\mathrm{Tg}$ and 13 non-Tg mice were obtained (data not shown) and these K-ras ${ }^{\mathrm{G} 12 \mathrm{D}} \mathrm{Tg}$ mice were used for analysis of K-ras-specific modulators by melphalan treatment in mouse model of lung cancer. K-ras ${ }^{\mathrm{G} 12 \mathrm{D}}$ transgenic mice were treated with or without melphalan for four weeks to assess the effects of melphalan on expression of K-ras biomarkers in lung tumorigenesis. Some of K-ras Tg mice showed abdominal adhesions caused by the intraperitoneal dosing vehicle of melphalan treatment. Non-
(A)
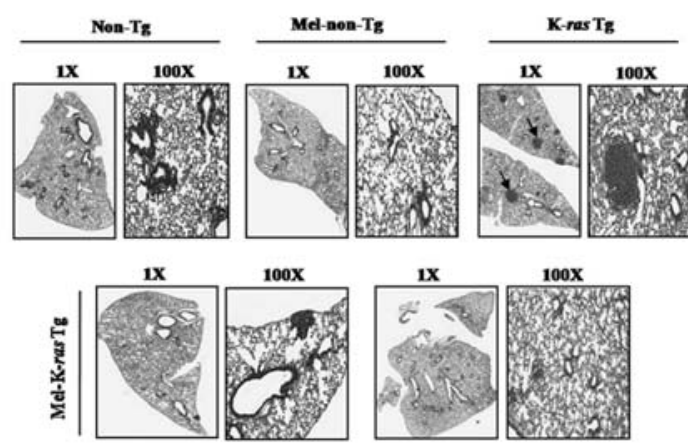

(B)

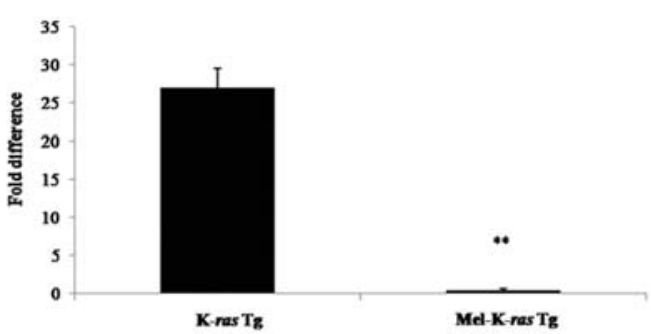

(C)

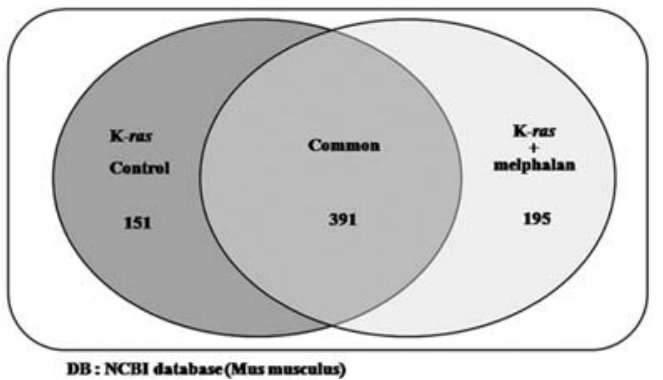

Figure 1. (A) Hematoxylin and eosin staining of lung tissues of non-Tg, melphalan-treated non- $\mathrm{Tg}, \mathrm{K}$-ras $\mathrm{Tg}$, and melphalan-treated K-ras mouse. (B) Real-time qPCR analysis of K-ras oncogene expression in K-ras $\mathrm{Tg}$ mice and melphalan-treated K-ras Tg mice. (C) Proteomics analysis of protein profiling of lung proteins modulated by melphalan in $\mathrm{K}$-ras $\mathrm{Tg}$ mice. The number of proteins changed significantly in K-ras transgenic lung tissues treated with or without melphalan. The 195 proteins representing for 42 unique mRNAs were deregulated at both time points in lung adenomas of $\mathrm{K}$-ras $\mathrm{Tg}$ mouse.

Tg mice did not develop adenoma and the size of adenomas was substantially decreased in melphalan-treated $\mathrm{K}$-ras $\mathrm{Tg}$ lungs compared to control K-ras Tg mice (Fig. 1A). Because the adenomas developed in a very small area of the whole lung tissues of K-ras mice and thus it is practically very difficult to purify RNAs from adenomas for comparing the transcript and protein profiles, whole lung tissues were used. To investigate the effect of melphalan on expression of $\mathrm{K}$-ras oncogene in $\mathrm{K}$-ras $\mathrm{Tg}$ mice, expression change of $\mathrm{K}$-ras oncogene was detected by real-time qPCR analysis between K-ras and melphalan-treated K-ras Tg mice. The expression of K-ras oncogene was dramatically enhanced in $\mathrm{Tg}$ mice compared to non-Tg mice (fold difference 1.0) and fully inhibited by treatment with melphalan (Fig. 1B).

Profiling analysis of proteins modulated by melphalan. Proteins modulated by melphalan in K-ras Tg mice were analyzed by LC-ESI-MS/MS. Total 436 proteins up-regulated and 301 proteins down-regulated by melphalan treatment 
Table II. Proteomics analysis of lung proteins modulated by melphalan in K-ras Tg mice.

\begin{tabular}{lrr}
\hline \multirow{2}{*}{ Fold change } & \multicolumn{2}{c}{ Mus musculus DB } \\
\cline { 2 - 3 } & Up-regulated & Down-regulated \\
\hline 3 & 173 & 117 \\
$3-5$ & 29 & 12 \\
$5-9$ & 34 & 15 \\
$>10$ & 200 & 157 \\
Total & 436 & 301 \\
\hline
\end{tabular}

Data files were extracted using Spectrum Mill Data Extractor with the parameters of $\left[\mathrm{MH}^{+}\right] 600-40000$ and minimum signal-to-noise $(\mathrm{S} / \mathrm{N}) 25$. Searches were carried out against the human NCBInr database in both forward and reverse directions using the Spectrum Mill program (Agilent Technologies) with the following parameters: specific to trypsin with two missed cleavage; $\pm 2.5 \mathrm{Da}$ precursor-ion tolerance; and $\pm 0.7 \mathrm{Da}$ fragment-ion tolerance. The initial results were auto-validated as described in the Methods section. were classified by fold change, as shown in Table II. The proteins expressed in K-ras Tg mice (151 proteins) and melphalan-treated K-ras Tg mice (195 proteins) were classified based on the cellular component (Fig. 1C). Proteins upand down-regulated by melphalan in lung tissue of $\mathrm{K}$-ras $\mathrm{Tg}$ mice are listed in Table III. K-ras-specific proteins modulated by melphalan were annotated by categories based on the GO (http://www.geneontology.org/): biological process and cellular component using the house-made FindGo program as shown in Fig. 2. With regard to both up- and down-regulated proteins, the proteins were classified into immune system, response, metabolism, transport and localization, organization and biogenesis, cell cycle and cytokinesis, cell adhesion, proteolysis, translation/transcription and nucleotide, protein modification, and unknown. Among the above criteria, the proportion of proteins involved in metabolism was large in both $\mathrm{K}$-ras $\mathrm{Tg}$ and melphalantreated K-ras Tg mice (Fig. 2).

Identification of K-ras-specific markers modulated by melphalan in K-ras transgenic lungs. To investigate the effect of
A)

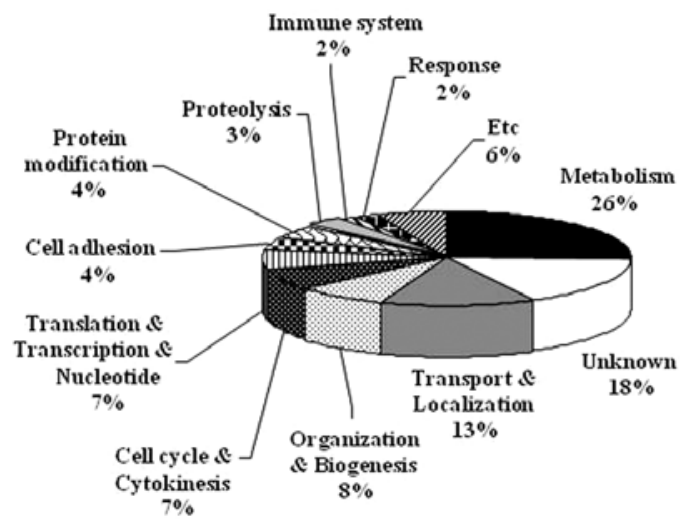

B)

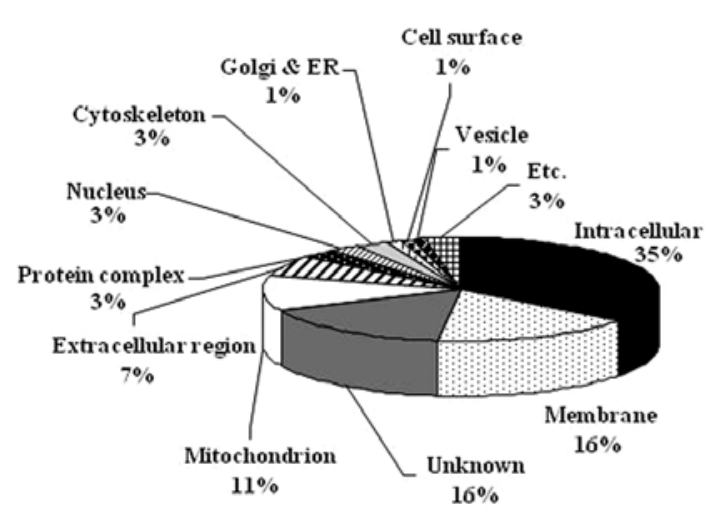

\section{Melphalan treated K-ras}

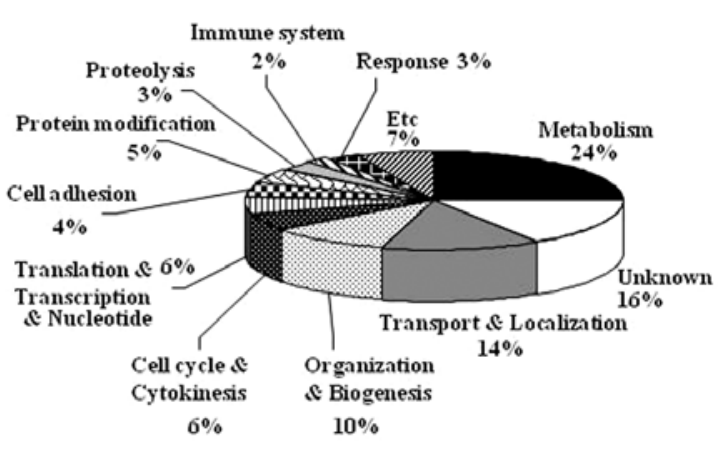

Melphalan treated K-ras

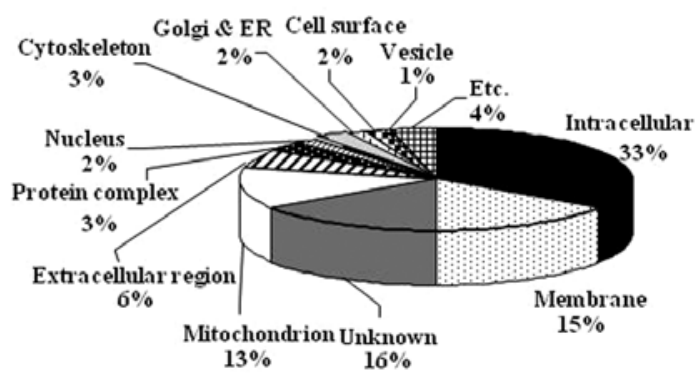

Figure 2. Pie chart showing proteins expressed in K-ras Tg control mice and melphalan-treated K-ras Tg mice based on the biological process (A) and the cellular component (B) as described in Methods section. 
Table III. A, Proteins up-regulated in melphalan-treated K-ras Tg mice.

\begin{tabular}{|c|c|c|c|}
\hline Protein name & Accession no. & $\begin{array}{c}\text { Peptides } \\
\text { unique no. }\end{array}$ & $\begin{array}{l}\text { Mel-K-ras/ } \\
\text { K-ras cont. }\end{array}$ \\
\hline \multicolumn{4}{|l|}{ Up-regulated } \\
\hline Tropomyosin 4 & 47894398 & 5 & 1.17 \\
\hline Diaphorase 1 (Cyb5r3) & 19745150 & 6 & 1.24 \\
\hline Rho GDP dissociation inhibitor (48) $\alpha$ & 31982030 & 5 & 1.65 \\
\hline Vimentin & 55408 & 21 & 1.68 \\
\hline RAB7, member RAS oncogene family & 6679599 & 4 & 1.72 \\
\hline Cofilin 1 , non-muscle & 6680924 & 5 & 1.88 \\
\hline RAB5C, member RAS oncogene family & 113866024 & 3 & 1.89 \\
\hline RAB1B, member RAS oncogene family & 21313162 & 6 & 2.40 \\
\hline Myosin, light polypeptide 7 , regulatory & 114326499 & 4 & 6.29 \\
\hline Proteasome (prosome, macropain) subunit, $\alpha$ type 1 & 33563282 & 2 & 6.58 \\
\hline Manganese superoxide dismutase & 53450 & 2 & 6.87 \\
\hline AHNAK nucleoprotein isoform 1 & 61743961 & 26 & 7.69 \\
\hline Aldose reductase & 786001 & 2 & 7.86 \\
\hline Transthyretin & 7305599 & 2 & 8.93 \\
\hline Carbonic anhydrase 3 & 31982861 & 9 & 11.13 \\
\hline Serine (or cysteine) proteinase inhibitor, clade A, member 6 & 6680856 & 6 & 160.92 \\
\hline Creatine kinase, muscle & 6671762 & 5 & \\
\hline ATP synthase, $\mathrm{H}^{+}$transporting, mitochondrial F0 complex, subunit $\mathrm{d}$ & 21313679 & 4 & \\
\hline Ubiquitin C & 21070950 & 4 & $>10^{4}$ \\
\hline Cytochrome b-5 & 13385268 & 2 & \\
\hline Peroxiredoxin V & 6644338 & 2 & \\
\hline Protein disulfide isomerase associated 4 & 86198316 & 2 & \\
\hline Myosin, light polypeptide 4 & 31981605 & 4 & \\
\hline Muscle glycogen phosphorylase & 6755256 & 2 & \\
\hline Calponin 2 & 6680952 & 2 & \\
\hline Caldesmon 1 & 21704156 & 1 & \\
\hline Laminin 32 & 31982223 & 1 & \\
\hline Caveolin 2 & 18702317 & 1 & \\
\hline Muscle-specific $\beta 1$ integrin binding protein & 110625739 & 1 & \\
\hline Capping protein (actin filament) muscle $\mathrm{Z}$-line, $B$ isoform a & 83649737 & 1 & $>10^{4}$ \\
\hline $\mathrm{K}$-ras cellular oncogene & 1213018 & 1 & \\
\hline Proteasome (prosome, macropain) subunit, $ß$ type 5 & 15126628 & 1 & \\
\hline Troponin I, cardiac & 6678393 & 1 & \\
\hline Myosin, light polypeptide 4 & 31981605 & 1 & \\
\hline Myosin, light polypeptide 3 & 33563264 & 1 & \\
\hline Muscle glycogen phosphorylase & 6755256 & 1 & \\
\hline Electron transferring flavoprotein, $\beta$ polypeptide & 38142460 & 1 & \\
\hline
\end{tabular}

List of the proteins, identified by their accession number and peptide unique number, up-regulated more than 1-104-fold in melphlan-K-ras $\mathrm{Tg}$ vs K-ras Tg mice. Because several intensities of K-ras are 0, fold changes of data up-regulated in melphalan-treated K-ras Tg, represent the ratio of melphlan-K-ras vs K-ras as $>10^{4}$.

B, Proteins up-regulated in melphalan-treated K-ras Tg mice.

\begin{tabular}{lcc}
\hline Protein name & Accession no. & $\begin{array}{c}\text { Peptides } \\
\text { unique no. }\end{array}$ \\
K-ras cont./ \\
mel-K-ras
\end{tabular}

Down-regulated

Destrin

Eukaryotic translation elongation factor $1 \delta$ isoform a

Mitogen-activated protein kinase 3

$21489933 \quad 3$

Exportin 1, CRM1 homolog 
Table III. B, Continued.

\begin{tabular}{|c|c|c|c|}
\hline Protein name & Accession no. & $\begin{array}{c}\text { Peptides } \\
\text { unique no. }\end{array}$ & $\begin{array}{c}\mathrm{K} \text {-ras cont./ } \\
\text { mel-K-ras }\end{array}$ \\
\hline \multicolumn{4}{|l|}{ Down-regulated } \\
\hline $\begin{array}{l}\text { Cell surface glycoprotein MUC18 precursor } \\
\text { (melanoma-associated antigen MUC18) } \\
\text { (melanoma cell adhesion molecule) (gicerin) }\end{array}$ & 81915013 & 2 & \\
\hline Sideroflexin 3 & 16716499 & 2 & \\
\hline Proteasome (prosome, macropain) subunit, $\beta$ type 10 & 13435741 & 1 & $>10^{4}$ \\
\hline Splicing factor $3 \mathrm{~b}$, subunit 3 & 19527174 & 1 & \\
\hline Coronin, actin binding protein $1 \mathrm{~A}$ & 31418362 & 1 & \\
\hline Ras homolog gene family, member $\mathrm{G}$ & 9625037 & 1 & \\
\hline Karyopherin (importin) $ß 1$ & 88014720 & 1 & \\
\hline Ribosomal protein L4 & 30794450 & 1 & \\
\hline Tropomodulin 2 & 6934242 & 1 & \\
\hline Ras-interacting protein 1 (Rain) & 81174999 & 1 & \\
\hline Toll-like receptor 12 & 45430001 & 1 & \\
\hline Immunoglobulin $\gamma 1$ heavy chain & 21304450 & 1 & \\
\hline Coronin, actin binding protein $1 \mathrm{~B}$ & 6753494 & 1 & \\
\hline Sideroflexin 3 & 16716499 & 1 & \\
\hline Galactokinase 1 & 29747831 & 1 & \\
\hline H3 histone, family 2 isoform 2 & 30061401 & 3 & 72.25 \\
\hline mKIAA1620 protein & 50511025 & 8 & 29.00 \\
\hline Splicing factor, arginine/serine-rich 7 & 71162370 & 2 & 15.74 \\
\hline Serum amyloid P-component precursor (SAP) & 134198 & 1 & 10.06 \\
\hline Transmembrane protein 109 & 19527378 & 1 & 9.91 \\
\hline Galectin-3 (galactose-specific lectin 3) & 126679 & 1 & 8.98 \\
\hline Actin-related protein $2 / 3$ complex, subunit 5 & 13385866 & 1 & 9.92 \\
\hline Epoxide hydrolase 1, microsomal & 6753762 & 4 & 8.75 \\
\hline Myeloid bactenecin (F1) & 2071985 & 1 & 7.96 \\
\hline Procollagen, type VI, $\alpha 1$ & 6753484 & 3 & 7.74 \\
\hline $\begin{array}{l}\text { Serine (or cysteine) proteinase inhibitor, clade } \mathrm{B} \text {, } \\
\text { member } 6 \mathrm{a}\end{array}$ & 6678097 & 5 & 6.53 \\
\hline Ribosomal protein L19 & 6677773 & 1 & 6.14 \\
\hline Glutamate dehydrogenase 1 & 6680027 & 5 & 6.05 \\
\hline Keratin 76 & 85701680 & 1 & 5.92 \\
\hline Keratin complex 2, basic, gene 17 & 46275808 & 1 & 5.48 \\
\hline Histone $1, \mathrm{H} 1 \mathrm{~d}$ & 34328365 & 1 & 5.40 \\
\hline Heat shock protein $1 ß$ & 40556608 & 17 & 5.33 \\
\hline Eukaryotic translation elongation factor $1 \gamma$ & 110625979 & 2 & 4.76 \\
\hline Catalase & 115704 & 8 & 3.51 \\
\hline Myo1b protein & 32452000 & 3 & 3.10 \\
\hline Calnexin & 6671664 & 1 & 2.80 \\
\hline Ubiquitin-activating enzyme E1, Chr X & 6678483 & 8 & 2.63 \\
\hline Eukaryotic translation elongation factor $1 \alpha 1$ & 13278382 & 8 & 2.55 \\
\hline Gelsolin & 28916693 & 8 & 2.17 \\
\hline Integrin $\alpha 1$ & 84370023 & 2 & 1.93 \\
\hline Annexin A4 & 7304889 & 8 & 1.52 \\
\hline
\end{tabular}

List of the proteins, identified by their accession number and peptide unique number, down-regulated more than 1-104-fold in melphlan-K-ras $\mathrm{Tg}$ vs K-ras Tg mice. Because several intensities of melphalan-K-ras are 0, fold changes of data down-regulated in melphalan-treated K-ras $\mathrm{Tg}$, represent the ratio of K-ras vs mel-K-ras as $>10^{4}$. 
(A)
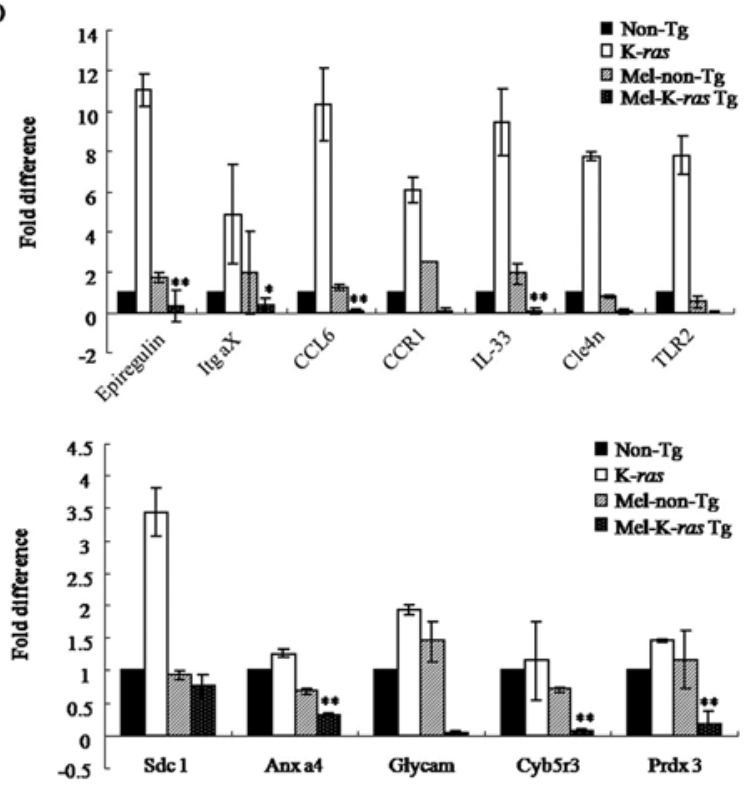

(B)

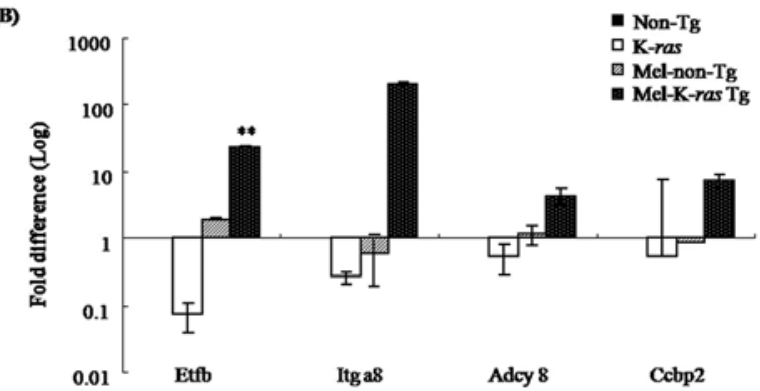

(C)

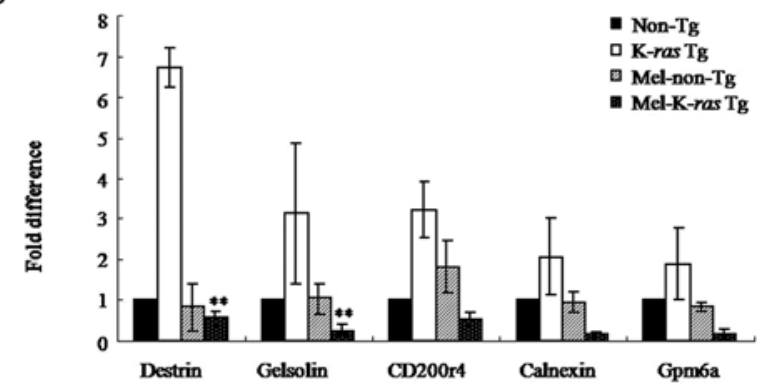

(D)

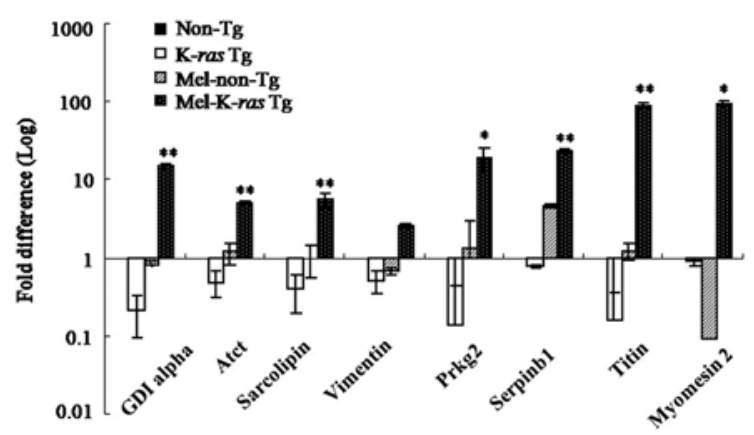

Figure 3. The quantitative PCR analysis of expressed genes regulated by $\mathrm{K}$-ras in K-ras Tg mice treated with melphalan. K-ras modulators identified in the previous study were (A) down-regulated and (B) up-regulated by melphalan. New identified K-ras modulators were (C) down-regulated and (D) up-regulated by melphalan in K-ras Tg mice. The gene expression level obtained from real-time qPCR was normalized by $\beta$-actin. The respective fold changes were expressed by comparing with the values of non-Tg mice which were set as 1 . Data are mean $\pm \mathrm{SD}$ of three independent experiments, each performed in triplicates. ${ }^{*} \mathrm{P}<0.05$ and ${ }^{* *} \mathrm{P}<0.01$ indicate statistical significance.

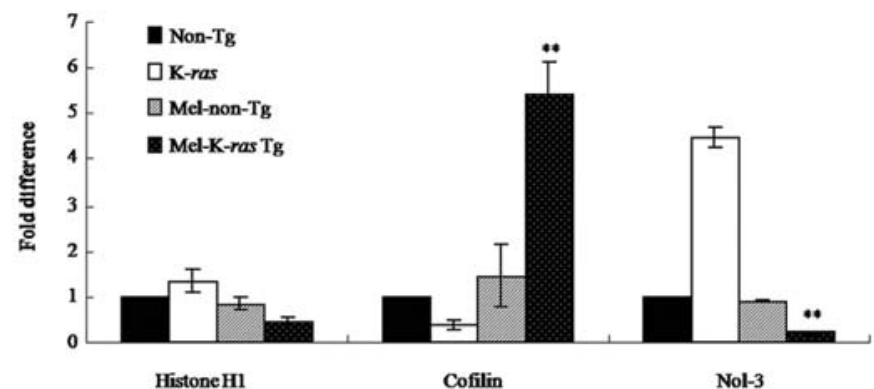

Figure 4. The effect of a genotoxic carcinogen melphalan on common predictive biomarkers in the lung tissues of K-ras $\mathrm{Tg}$ mice. ${ }^{* *} \mathrm{P}<0.01$ indicates statistical significance.

melphalan on the expressions of K-ras modulators in lung tissues of K-ras Tg mice, we measured the expression levels of modulators regulated by melphalan in K-ras Tg mice using real-time qPCR with $1 X$ iQ SYBR-Green Supermix (Bio-Rad). Non-Tg mice were used as negative control, and $\mathrm{K}$-ras $\mathrm{Tg}$ mice and melphalan-treated non- $\mathrm{Tg}$ were used as positive control versus melphalan treatment of K-ras $\mathrm{Tg}$ mice in this experiment. With regard to genes up- and downregulated by K-ras oncogene in a previous study (9), the expression patterns of these genes were recovered by melphalan treatment (Fig. 3A-D). The proteins considered to be related to cancer cell adhesion, signal transduction, migration and cancer development were selected by proteomics analysis. In addition to the gene profiling, new proteins such as vimentin, troponin $\mathrm{T} 2$ cardiac, destrin, calnexin, gelsolin, titin, actin $\alpha$ cardiac (atct), protein kinase cGMP-dependent type II (Prkg2), serine peptidase inhibitor clade B, member 1 (Serpinb1), GDI $\alpha$, myomesin 2, and sarcolipin were selected and their expressions were evaluated by real-time qPCR.

The effect of melphalan on common biomarkers in the lung tissues of K-ras Tg mice. Histone H1, cofilin-1 and nucleolar protein 3 (nol-3) known as predictive markers in tumor $(19,20)$ were assessed in order to prove the effect of melphalan on expressions of K-ras predictive markers in $\mathrm{K}$-ras $\mathrm{Tg}$ mice. Histone $\mathrm{H} 1$ and nol-3 $(\mathrm{P}<0.01)$ were down-regulated by melphalan in K-ras Tg mice. Expression level of cofilin-1 was significantly increased in $\mathrm{K}-\mathrm{ras} \mathrm{Tg}$ mouse lungs treated with melphalan $(\mathrm{P}<0.01)$ (Fig. 4).

\section{Discussion}

Ras family is involved in regulation of cell proliferation and development and activated by somatic point mutations in various human tumors $(21,22)$ as well as in experimental animal models (23). K-ras oncogenes have frequently been observed in mouse or human lung tumors (24-26). Activating mutation in the K-ras oncogenes may accelerate more progression of adenomas and malignant tumors by various genotoxic carcinogens in transgenic mouse models and tumors $(25,27)$. However, anti-cancer or DNA-damaging agents or genotoxic carcinogens such as melphalan induce apoptosis in carcinoma cells $(28,29)$. It is necessary to monitor the modulating effects of melphalan treatment on 
$\mathrm{K}$-ras $\mathrm{Tg}$ mice in order to prove $\mathrm{K}$-ras-modulated biomarkers identified by a previous study (9). When the genotoxic carcinogen melphalan was administered into K-ras transgenic mice, melphalan recovered the altered expression of K-ras modulators for lung tumorigenesis in K-ras $\mathrm{Tg}$ mice. On the basis of our previous work (9) and in order to elucidate whether melphalan would regulate progression of adenomas and malignant tumors as a genotoxic carcinogen or modulate the K-ras-induced biomarkers as an anti-cancer agent in transgenic mouse models, we investigated the effect of melphalan on expression of K-ras modulators in K-ras ${ }^{\mathrm{G} 12 \mathrm{D}}$ $\mathrm{Tg}$ mice by proteomics analysis and real-time qPCR. Several $\mathrm{K}$-ras or non-Tg mice treated with melphalan represented abdominal adhesion caused by intraperitoneal dosing vehicle. Hematoxylin and eosin staining of lung tissues revealed that multifocal papillary adenomas were decreased or inhibited only in K-ras transgenic lung tissues with four-week treatment of melphalan. K-ras oncogene expression was suppressed by melphalan treatment in K-ras Tg mice. In addition, melphalan down-regulated the expression levels of $\mathrm{K}$-ras-induced genes involved in the molecular function (nucleic acid binding, receptor, metabolism and catabolism, transport and localization, development and signaling molecule) and pathway (angiogenesis, inflammation mediated by chemokine and cytokine signaling, proliferation, cell cycle and apoptosis). These facts revealed that K-ras modulators in $\mathrm{K}$-ras $\mathrm{Tg}$ mice are more susceptible to melphalan than control non-Tg mice. The expressions of several genes for biological processes showing a decreasing tendency in $\mathrm{K}$-ras $\mathrm{Tg}$ mice were also significantly recovered by melphalan treatment. Expressions of new genes such as calnexin, gelsolin, destrin, vimentin, CD200 receptor 4 (CD200r4) and titin (as shown in Table I) selected on the basis of proteomics data were analyzed by real-time qPCR in $\mathrm{K}-$ ras Tg lung tissues treated with melphalan. Myomesin 2, titin, sarcolipin and cardiac $\alpha$ actin, involved in cell adhesion and metastasis $(30,31)$, were up-regulated by melphalan. Type II cGMP-dependent protein kinase (Prkg2) significantly correlated with survival (32) was also increased by melphalan treatment and it could inhibit tumor progression toward lung metastasis formation by blocking the immunosuppressor mechanism triggered by agents that increase intracellular cAMP (33). Cardiac troponin T2 modulated by melphalan is involved in cardiopulmonary bypass (CPB)related inflammatory response (34). Mouse models have multiple CD200 receptors, expressed on cells of the monocyte/macrophage lineage and on T lymphocytes, including stimulatory and inhibitory receptors $(35,36)$. Expression level of vimentin which is methylated in most colorectal neoplasms but rarely in normal epithelia (37) was down-regulated by K-ras and recovered in melphalan-treated $\mathrm{K}$-ras $\mathrm{Tg}$ mice. Calnexin inhibits activity of proteins of tumor suppressor genes (38) and it was reported that expressions of calnexin and destrin were increased in A549 malignant lung epithelial cancer cells (39). Expressions of these calnexin and destrin were also down-regulated by melphalan. K-ras-induced gelsolin expression was suppressed by melphalan compared to $\mathrm{K}$-ras $\mathrm{Tg}$. In the case of rasH2 mice, genes such as gelsolin, and amphiregulin that were commonly altered in N-ethyl-N-nitrosourea or urethane-

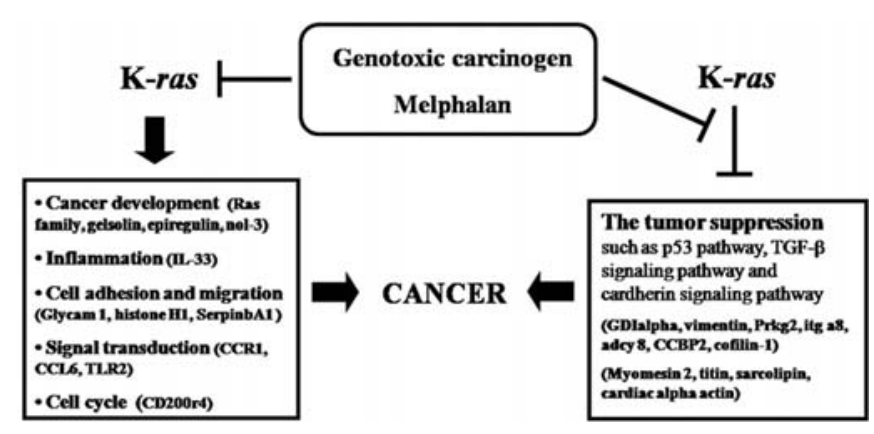

Figure 5. The schematic diagram for the effect of melphalan on cancer development by K-ras oncogene. Oncogenic K-ras triggers progression of cancer, by inducing markers related to cancer development and inflammation, signal transduction, cell adhesion, migration and cell cycle. Furthermore, K-ras inhibits tumor suppression, such as p53 pathway, resulting in carcinogenesis. Melphalan suppresses cancer progression by K-ras and modulates expression of K-ras-specific biomarkers, inhibiting K-ras expression in lung tissues of K-ras Tg mice.

induced adenomas may contribute to the development of tumors in rasH2 mice (40). In addition, serine peptidase inhibitor clade B, member 1 (Serpinb1) and GDI $\alpha$ were increased while expressions of glycoprotein m6a (gpm6a) and destrin induced by K-ras were decreased in lung tissues of K-ras Tg mice treated with melphalan. SerpinbA1 $(\alpha-1$ antitrypsin) is a marker of malignancy in insulinomas (41). In Rho GDI $\alpha$ knockdown cells, the rate of apoptotic cells increased when compared with the control small interfering RNA-transfected cells. In addition, treatment of cells with busulfan resulted in a further increased apoptotic rate, thus, expression of Rho GDI $\alpha$ influences the sensitivity of cells toward busulfan-induced cytotoxicity (42). Gpm6a is a neuron-specific membrane glycoprotein of the brain and possesses putative phosphorylation sites for protein kinase $\mathrm{C}$ (PKC) (43). Recent studies suggest that destrin is a significant regulator of various processes important for invasive phenotype of human colon cancer cells (44) and altered production, regulation or localization of this protein might lead to cognitive impairment, inflammation, infertility, immune deficiencies and other pathophysiological defects (45).

In the present study, we confirmed that the expression of genes known as common biomarkers in tumor is regulated by melphalan in K-ras Tg mice. Cofilin-1 was up-regulated while histone $\mathrm{H} 1$ and nol-3 were down-regulated by melphalan. Cofilin-1 is directly responsible for severing actin filaments and regulating actin polymerization and depolymerization during cell migration. Hence, cofilin is arguably the key effector to determine cell migration and invasion in tumor cells (46). $\mathrm{H} 1$ histones acting as basal repressors of transcription are essential for the generation of these condensed structures and for the initiation of the subsequent internucleosomal cleavage of DNA, and regulate cellular differentiation in tumor cells (47). Nucleolar protein-3 is known as an antiapoptotic gene and a marker for proliferation in lung and other cancer cells (20). We consider that melphalan suppresses cancer progression by K-ras and affects expression of K-ras modulators, inhibiting K-ras expression in lung tissues of $\mathrm{K}$-ras $\mathrm{Tg}$ mice. 
In conclusion, our results suggest that K-ras biomarkers playing an important role in carcinogenesis of K-ras mice are modulated by melphalan (Fig. 5). Oncogenic K-ras triggers progression of cancer by inducing markers related to cancer development and inflammation, signal transduction, cell adhesion, migration and cell cycle. Furthermore, K-ras oncogene inhibits tumor suppression such as p53 pathway, resulting in carcinogenesis (9). Melphalan treatment for 4 weeks modulated K-ras biomarkers in $\mathrm{K}$-ras $\mathrm{Tg}$ mice. The genes and proteins related to carcinogenesis or mediated by $\mathrm{K}$-ras in lung cancer are considered to be candidates for response to anti-cancer and genotoxic signals.

\section{Acknowledgments}

This research was supported by a grant from the National R\&D Program for Cancer Control, Ministry for Health, Welfare and Family Affairs, Republic of Korea (0920080) and in part from the basic program (2009-0072028) of the National Research Foundation (NRF) and a grant (09152644) from Korea Food \& Drug Administration. We thank Mr G.Y. Kang for his technical assistance. S.L. is supported in part by Seoul Scholarship Foundation and D.Y. is partially supported by Priority Research Centers Program (20090093824) through the National Research Foundation (NRF) of Korea.

\section{References}

1. Kerr KM: Pulmonary preinvasive neoplasia. J Clin Pathol 54: 257-271, 2001

2. You M, Candrian U, Maronpot RR, Stoner GD and Anderson MW: Activation of the $\mathrm{Ki}$-ras protooncogene in spontaneously occurring and chemically induced lung tumors of the strain A mouse. Proc Natl Acad Sci USA 86: 3070-3074, 1989.

3. Adjei AA: K-ras as a target for lung cancer therapy. J Thorac Oncol 3: S160-S163, 2008.

4. Rodenhuis $S$ and Slebos RJ: The ras oncogenes in human lung cancer. Am Rev Respir Dis 142: S27-S30, 1990.

5. Wislez M, Spencer ML, Izzo JG, et al: Inhibition of mammalian target of rapamycin reverses alveolar epithelial neoplasia induced by oncogenic K-ras. Cancer Res 65: 3226-3235, 2005.

6. Mills NE, Fishman CL, Rom WN, Dubin N and Jacobson DR: Increased prevalence of K-ras oncogene mutations in lung adenocarcinoma. Cancer Res 55: 1444-1447, 1995.

7. Kissil JL, Walmsley MJ, Hanlon L, et al: Requirement for Rac1 in a K-ras induced lung cancer in the mouse. Cancer Res 67: 8089-8094, 2007.

8. Kiyohara C, Yoshimasu K, Shirakawa T and Hopkin JM: Genetic polymorphisms and environmental risk of lung cancer: a review. Rev Environ Health 19: 15-38, 2004.

9. Lee S, Kang J, Cho M, et al: Profiling of transcripts and proteins modulated by K-ras oncogene in the lung tissues of K-ras transgenic mice by omics approaches. Int J Oncol 34: 161-172, 2009.

10. Souliotis VL, Dimopoulos MA, Episkopou HG, Kyrtopoulos SA and Sfikakis PP: Preferential in vivo DNA repair of melphalaninduced damage in human genes is greatly affected by the local chromatin structure. DNA Repair 5: 972-985, 2006.

11. Spanswick VJ, Craddock C, Sekhar M, et al: Repair of DNA interstrand crosslinks as a mechanism of clinical resistance to melphalan in multiple myeloma. Blood 100: 224-229, 2002.

12. Dimopoulos MA, Souliotis VL, Anagnostopoulos A, Papadimitriou C and Sfikakis PP: Extent of damage and repair in the p53 tumor-suppressor gene after treatment of myeloma patients with high-dose melphalan and autologous blood stemcell transplantation is individualized and may predict clinical outcome. J Clin Oncol 23: 4381-4389, 2005.

13. Keane MM, Ettenberg SA, Nau MM, Russell EK and Lipkowitz S: Chemotherapy augments TRAIL-induced apoptosis in breast cell lines. Cancer Res 59: 734-741, 1999.
14. de Palo GM, de Lena M, Di Re F, Luciani L, Valagussa P and Bonadonna G: Melphalan versus adriamycin in the treatment of advanced carcinoma of the ovary. Surg Gynecol Obstet 141: 899-902, 1975.

15. Kanclerz A and Chapman JD: The effectiveness of cis-platinum, cyclophosphamide and melphalan in treating disseminated tumor cells in mice. Clin Exp Metastasis 5: 199-211, 1987.

16. Wang AG, Moon HB, Lee MR, et al: Gender-dependent hepatic alterations in H-ras $12 \mathrm{~V}$ transgenic mice. J Hepatol 43: 836-844, 2005.

17. Frith $\mathrm{CH}$ and Ward JM: A morphologic classification of proliferative and neoplastic hepatic lesions in mice. J Environ Pathol Toxicol 3: 329-351, 1979.

18. Qian WJ, Liu T, Monroe ME, et al: Probability-based evaluation of peptide and protein identifications from tandem mass spectrometry and SEQUEST analysis: the human proteome. J Proteome Res 4: 53-62, 2005.

19. Keshamouni VG, Michailidis G, Grasso CS, et al: Differential protein expression profiling by iTRAQ-2DLC-MS/MS of lung cancer cells undergoing epithelial-mesenchymal transition reveals a migratory/invasive phenotype. J Proteome Res 5: 1143-1154, 2006.

20. Wang M, Qanungo S, Crow MT, Watanabe M and Nieminen AL: Apoptosis repressor with caspase recruitment domain (ARC) is expressed in cancer cells and localizes to nuclei. FEBS Lett 579: 2411-2415, 2005.

21. Wang XQ, Li H, Van Putten V, Winn RA, Heasley LE and Nemenoff RA: Oncogenic K-Ras regulates proliferation and cell junctions in lung epithelial cells through induction of cyclooxygenase- 2 and activation of metalloproteinase-9. Mol Biol Cell 20: 791-800, 2009.

22. Johnson L, Mercer K, Greenbaum D, et al: Somatic activation of the K-ras oncogene causes early onset lung cancer in mice. Nature 410: 1111-1116, 2001.

23. Wakamatsu N, Devereux TR, Hong HH and Sills RC: Overview of the molecular carcinogenesis of mouse lung tumor models of human lung cancer. Toxicol Pathol 35: 75-80, 2007.

24. Chen B, Johanson L, Wiest JS, Anderson MW and You M: The second intron of the $\mathrm{K}$-ras gene contains regulatory elements associated with mouse lung tumor susceptibility. Proc Natl Acad Sci USA 91: 1589-1593, 1994.

25. Devereux TR, Anderson MW and Belinsky SA: Role of ras protooncogene activation in the formation of spontaneous and nitrosamine-induced lung tumors in the resistant $\mathrm{C} 3 \mathrm{H}$ mouse. Carcinogenesis 12: 299-303, 1991.

26. Herzog CR, Lubet RA and You M: Genetic alterations in mouse lung tumors: implications for cancer chemoprevention. J Cell Biochem 28-29: 49-63, 1997.

27. Yamamoto S, Mitsumori K, Kodama Y, et al: Rapid induction of more malignant tumors by various genotoxic carcinogens in transgenic mice harboring a human prototype c-Ha-ras gene than in control non-transgenic mice. Carcinogenesis 17: 2455-2461, 1996.

28. Basu S, Ma R, Boyle PJ, et al: Apoptosis of human carcinoma cells in the presence of potential anti-cancer drugs: III. Treatment of Colo-205 and SKBR3 cells with: cis-platin, tamoxifen, melphalan, betulinic acid, L-PDMP, L-PPMP, and GD3 ganglioside. Glycoconj J 20: 563-577, 2004.

29. Kelner MJ, McMorris TC, Rojas RJ, Estes LA and Suthipinijtham P: Synergy of irofulven in combination with other DNA damaging agents: synergistic interaction with altretamine, alkylating, and platinum-derived agents in the MV522 lung tumor model. Cancer Chemother Pharmacol 63: 19-26, 2008.

30. Carothers AM, Javid SH, Moran AE, Hunt DH, Redston M and Bertagnolli MM: Deficient E-cadherin adhesion in C57BL/6JMin/ ${ }^{+}$mice is associated with increased tyrosine kinase activity and RhoA-dependent actomyosin contractility. Exp Cell Res 312: 387-400, 2006.

31. Camara-Pereira ES, Campos LM, Vannier-Santos MA, Mermelstein CS and Costa ML: Distribution of cytoskeletal and adhesion proteins in adult zebrafish skeletal muscle. Histol Histopathol 24: 187-196, 2009.

32. Ikehara M, Oshita F, Sekiyama A, et al: Genome-wide cDNA microarray screening to correlate gene expression profile with survival in patients with advanced lung cancer. Oncol Rep 11: 1041-1044, 2004.

33. Blaya C, Crespo J, Crespo A and Alino SF: Effect of the protein kinase inhibitors, 1-(5-isoquinolinylsulfonyl)-2-methylpiperazine $\mathrm{H}-7$ and $\mathrm{N}-(2-[$ methylamino]ethyl)-5-isoquinoline-sulfonamide $\mathrm{H}-8$ on Lewis lung carcinoma tumor progression. Eur J Pharmacol 354: 99-104, 1998. 
34. Liakopoulos OJ, Schmitto JD, Kazmaier S, et al: Cardiopulmonary and systemic effects of methylprednisolone in patients undergoing cardiac surgery. Ann Thorac Surg 84: 110-118; discussion 118-119, 2007.

35. Barclay AN, Wright GJ, Brooke G and Brown MH: CD200 and membrane protein interactions in the control of myeloid cells. Trends Immunol 23: 285-290, 2002.

36. Wright GJ, Cherwinski H, Foster-Cuevas M, et al: Characterization of the CD200 receptor family in mice and humans and their interactions with CD200. J Immunol 171: 3034-3046, 2003.

37. Zou H, Harrington JJ, Shire AM, et al: Highly methylated genes in colorectal neoplasia: implications for screening. Cancer Epidemiol Biomarkers Prev 16: 2686-2696, 2007.

38. Landolph JR, Verma A, Ramnath J and Clemens F: Molecular biology of deregulated gene expression in transformed $\mathrm{C} 3 \mathrm{H} /$ 10T1/2 mouse embryo cell lines induced by specific insoluble carcinogenic nickel compounds. Environ Health Perspect 110 (Suppl. 5): S845-S850, 2002.

39. Chang JW, Jeon HB, Lee JH, et al: Augmented expression of peroxiredoxin I in lung cancer. Biochem Biophys Res Commun 289: 507-512, 2001.

40. Okamura M, Unami A, Moto M, et al: The possible mechanism of enhanced carcinogenesis induced by genotoxic carcinogens in rasH2 mice. Cancer Lett 245: 321-330, 2007.

41. de Sa SV, Correa-Giannella ML, Machado MC, et al: Serpin peptidase inhibitor clade A member 1 as a potential marker for malignancy in insulinomas. Clin Cancer Res 13: 5322-5330, 2007.
42. Reimer J, Bien S, Sonnemann J, et al: Reduced expression of Rho guanine nucleotide dissociation inhibitor-alpha modulates the cytotoxic effect of busulfan in HEK293 cells. Anticancer Drugs 18: 333-340, 2007.

43. Mukobata S, Hibino T, Sugiyama A, et al: M6a acts as a nerve growth factor-gated $\mathrm{Ca}(2+)$ channel in neuronal differentiation. Biochem Biophys Res Commun 297: 722-728, 2002.

44. Estornes Y, Gay F, Gevrey JC, et al: Differential involvement of destrin and cofilin-1 in the control of invasive properties of Isreco1 human colon cancer cells. Int J Cancer 121: 2162-2171, 2007.

45. Bamburg JR and Wiggan OP: ADF/cofilin and actin dynamics in disease. Trends Cell Biol 12: 598-605, 2002.

46. Wang W, Mouneimne G, Sidani M, et al: The activity status of cofilin is directly related to invasion, intravasation, and metastasis of mammary tumors. J Cell Biol 173: 395-404, 2006.

47. Kratzmeier M, Albig W, Meergans T and Doenecke D: Changes in the protein pattern of $\mathrm{H} 1$ histones associated with apoptotic DNA fragmentation. Biochem J 337: 319-327, 1999.

48. Pandey J, Umphress SM, Kang Y, et al: Modulation of tumor induction and progression of oncogenic K-ras-positive tumors in the presence of TGF- b1 haploinsufficiency. Carcinogenesis 28: 2589-2596, 2007. 